\title{
Is the undoped cuprate a simple antiferromagnet?
}

\author{
Giant thermal Hall conductivity from neutral excitations in the pseudogap \\ phase of cuprates \\ Authors: G. Grissonnanche et al \\ arXiv: 1901.03104
}

\section{Recommended with a Commentary by Dung-Hai Lee, U.C. Berkeley}

In the field of copper-oxide high-temperature superconductivity, it is commonly thought that the antiferromagnetism in the undoped parent compound is an open-and-shut chapter. Theoretical descriptions using the 2D non-linear sigma model[1] or spin 1/2 Heisenberg model[2] quantitatively agree with the experimental data. For example, the zero temperature Neel order parameter of the Heisenberg model agrees with the measured value when the temperature is much lower than the antiferromagnetic ordering temperature $T_{N}$. In addition, the temperature dependent correlation length predicted by the non-linear sigma or the Heisenberg model well describe the measured correlation length for $T>T_{N}$. The agreement also applies to the spin-wave dispersion. Moreover, more subtle experimental details can also be explained after the inclusion of additional weak perturbations, e.g., the coupling between the adjacent copper-oxygen planes, the Dzyaloshinskii-Moriya interaction, and the anisotropy in the in-plane Heisenberg exchange. On top of this, the spin $1 / 2$ Heisenberg model naturally arises from the superexchange in strongly-correlated microscopic models, such as the Hubbard model, when there is one electron per lattice site. Thus it is fair to believe that the physics of the parent compound is iwell understood.

The paper by Grissonnanche et al puts a question mark on the above sentiment. By measuring the thermal conductivity, in particular, the thermal Hall conductivity $\kappa_{x y}$, they discovered an unexpected phenomenon which seems to require the existence of a yet unknown type of gapless excitations.

The experiment is done on a number of copper-oxide compounds (including $\mathrm{La}_{2-x} \mathrm{Sr}_{x} \mathrm{CuO}_{4}$, $\left.\mathrm{La}_{1.6-x} \mathrm{Nd}_{0.4} \mathrm{Sr}_{x} \mathrm{CuO}_{4}, \mathrm{Bi}_{2} \mathrm{Sr}_{2-x} \mathrm{La}_{x} \mathrm{CuO}_{6+\delta}, \mathrm{La}_{1.8-x} \mathrm{Eu}_{0.2} \mathrm{Sr}_{x} \mathrm{CuO}_{4}\right)$ whose doping $(p)$ lies outside that under the green rectangle in figure $1(\mathrm{~b})$. The measurements are performed in the normal state where the superconductivity is suppressed by a magnetic field. While to the right of the so-called quantum critical doping, $p^{*}$, the thermal Hall conductivity can be attributed to the thermal conduction of charged carriers via the Wiedemann-Franz law in the entire temperature range. However, in the doping range to the left of $p^{*}, \kappa_{x y} / T$ first changes 
(a)

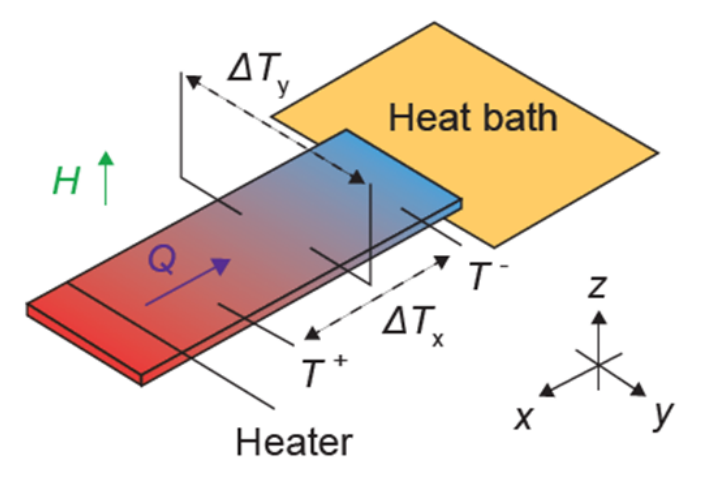

(b)

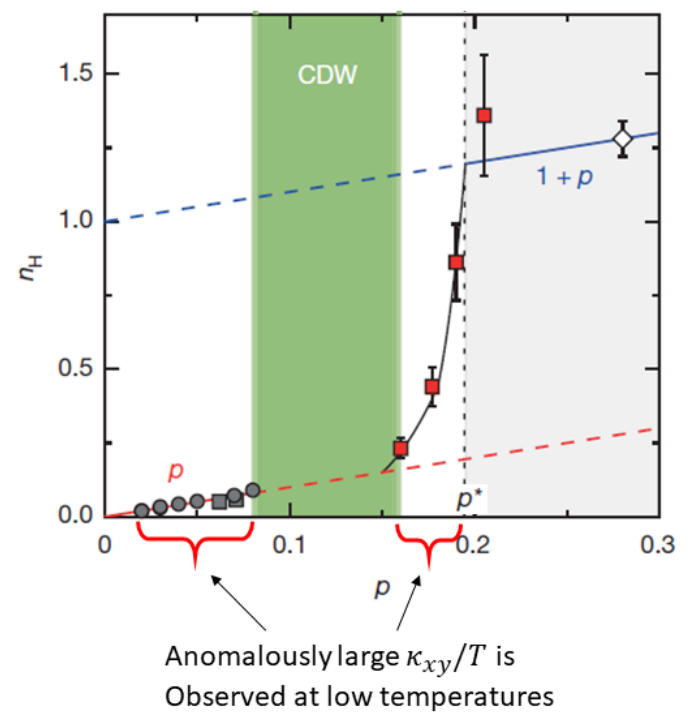

Figure 1: (a) the set up for the thermal Hall conductivity measurement. (b) The Hall number versus doping in Ref.[3] . The green rectangle covers the doping range where an extra complication, namely, charge order, has been observed.

sign and grows in magnitude as a function of decreasing temperature. At low temperatures its sign becomes opposite to that observed for $p>p^{*}$, hence is inexplicable in terms of charge carriers.

A particularly interesting result is obtained from $\mathrm{La}_{2-0.06} \mathrm{Sr}_{0.06} \mathrm{CuO}_{4}$. This sample sits right outside the antiferromagnetic ordered phase. Because at such low doping level $(\mathrm{x}=0.06)$ the superconducting $T_{c}$ is only $5 \mathrm{~K}$, a weak magnetic field suppresses superconductivity and so the thermal Hall measurement can be extended to low magnetic fields. The result, shown as the red curve in figure 2(a), exhibits a striking monotonic increase of $\kappa_{x y} / T$ with the magnetic field, somewhat similar to the behavior of the electric Hall conductivity of a metal. Moreover, under a strong magnetic field, the observed magnitude of $\kappa_{x y}$ per copper-oxide plane is comparable to the quantum of thermal conductance. In contrast, the longitudinal thermal conductance $\kappa_{x x}$ is found to be independent of the magnetic field (figure 2(a) blue line).

Interestingly, at a magnetic field of $15 \mathrm{~T}$, the undoped $\mathrm{La}_{2} \mathrm{CuO}_{4}$ shows a very similar temperature dependence of $\kappa_{x y} / T$ as the $\mathrm{x}=0.06$ doped $\mathrm{La}_{2-x} \mathrm{Sr}_{x} \mathrm{CuO}_{4}$. It suggests the same thermal conduction process is at work, and perhaps the $\kappa_{x y} / T$ of the undoped sample will also show a similar magnetic field dependence. (The field dependence of $\kappa_{x y} / T$ for $\mathrm{La}_{2} \mathrm{CuO}_{4}$ did not appear in the paper.) 
(a)

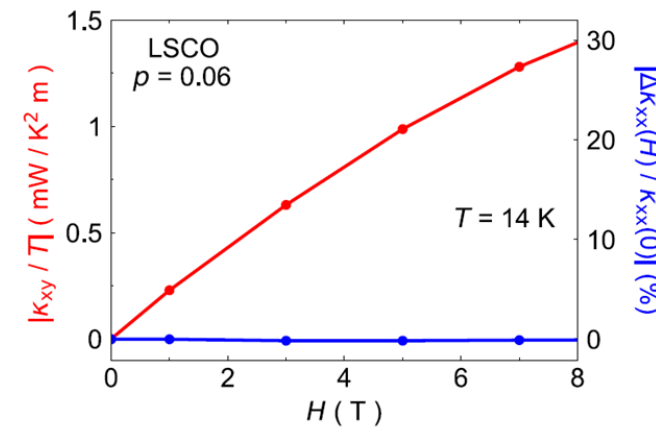

(b)

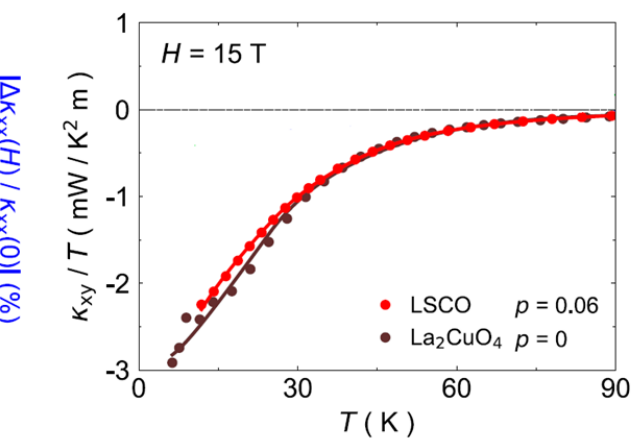

Figure 2: (a) The red curve corresponds to $\kappa_{x y} / T$, and the blue line illustrates the field dependence of $\kappa_{x x}$. (b) A comparison between the temperature dependence of $\kappa_{x y} / T$ associated with the $\mathrm{x}=0$ and $\mathrm{x}=0.06$ doping of $\mathrm{La}_{2-x} \mathrm{Sr}_{x} \mathrm{CuO}_{4}$.

An equally important fact is that similar thermal Hall conductance is observed for samples which do not show antiferromagnetic order. Indeed, for doping range outside that covered under the green rectangle in figure 1(a) similar $\kappa_{x y} / T$ is observed in a strong magnetic field and at temperatures where the superconductivity is absent. Very interestingly, it is precisely in this doping range earlier Hall coefficient measurement has determined the carrier density to be $p$, namely, the doping concentration. This is in marked contrast with the doping range of $p>p^{*}$ where the carrier density is $1+p[?]$. The $1+p$ is consistent with the value deduced from angle-resolved photoemission spectroscopy by subtracting the area enclosed by the hole-like Fermi surface from that of the first Brillouin zone. Compare $p$ and $1+p$ the missing 1 can be attributed to $1 / 2$ of the Brillouin zone area. This can be explained if the translation symmetry is spontaneously broken and the real space unit cell is doubled. But in this case, it is unclear whether the translation symmetry is really broken, and if it is, what kind of order is causing it. The unusual thermal Hall conductivity is observed when the missing Brillouin zone area occurs.

The question is what is the origin of the observed thermal Hall conduction? This is certainly not expected by the Heisenberg model. Under ordinary circumstances, aside from charge carriers, thermal conduction can be caused by magnons (the Goldstone mode associated with the magnetic order) and phonons. Grissonnanche et al considered them, and rule out the magnons by noting that the unusual $\kappa_{x y} / T$ exists in samples where there is no antiferromagnetic order (at least in zero magnetic field). They also argue against the phonons because the usual mechanism which causes phonon thermal Hall conductivity, namely phonon 
skew scattering, is very weak in the cuprates. Their conclusion agrees with the theoretical analysis performed in Ref.[4]. Grissonnanche et al also noted that the maximum magnitude of thermal Hall conductance they observe is similar to that observed in RuCl3 recently[5]. The latter data shows a quantized thermal Hall conductance consistent with that due to Chiral Majorana fermion edge modes.

Thus Grissonnanche et al's data reopens a closed chapter. If the experiment on the undoped cuprates stands scrutiny it is possible that the antiferromagnetic ordered insulating parent compound of the cuprates is not as simple as we thought. A number of theoretical works have already appeared on this subject $[4,6,7,8]$.

\section{References}

[1] S. Chakravarty, B. I. Halperin, and D. Nelson, Phys. Rev. Lett. 60, 1057 (1988); S. Chakravarty, B. I. Halperin, and D. Nelson, Phys. Rev. 8 39, 2344 (1989).

[2] For a review see, e.g., Efstratios Manousakis, Rev. Mod. Phys. 63,1, (1991).

[3] S. Badoux et al, Nature 531, 210 (2016).

[4] J. H. Han, J. -H. Park, and P. A. Lee, arXiv: 1903.01125 (2019).

[5] Y. Kasahara et al, Nature 559, 227 (2018).

[6] R. Samajdar et al, arXiv: 1812.08792 (2018).

[7] S. Chatterjee et al, arXiv:1903.01992 (2019).

[8] Z-X Li and D.-H. Lee arXiv:1905.04248 (2019). 